\title{
Sequelae of diaphyseal and supracondylar femoral fractures
}

\author{
Federico Chiodini', Luca Busnelli ${ }^{2}$ \\ 'UO Ortopedia e Traumatologia, Ospedale di Legnano, ASST Ovest Milanese, Italy; \\ ${ }^{2}$ Scuola di Specializzazione in Ortopedia e Traumatologia, Università di Cagliari, Italy
}

Received: November 28, 2019

Accepted: January 10, 2020

\section{Correspondence}

Federico Chiodini

UO Ortopedia e Traumatologia, Ospedale di Legnano, ASST Ovest Milanese, via Papa Giovanni Paolo II, 20025 Legnano (MI), Italy

E-mail: federico.chiodini@asst-ovestmi.it

Conflict of interest

The Authors declare no conflict of interest

How to cite this article: Chiodini F, Busnelli L. Sequelae of diaphyseal and supracondylar femoral fractures. Lo Scalpello 2020;34:56-62. https://doi.org/10.36149/0390-5276-009

(C) Ortopedici Traumatologi Ospedalieri d'Italia (O.T.O.D.i.) 2020

\section{(c) (i) $(-)$}

This is an open access article distributed in accordance with the CC-BY-NC-ND (Creative Commons Attribution-NonCommercial-NoDerivatives 4.0 International) license. The article can be used by giving appropriate credit and mentioning the license, but only for non-commercial purposes and only in the original version. For further information: https://creativecommons.org/licenses/by-nc-nd/4.0/deed.en

\section{SUMmarY}

Complications after surgical treatment of femoral diaphyseal and supracondylar fractures can occur. Adequate planning and knowledge of the principles of osteosynthesis can help to avoid predictable sequelae.

Key words: diaphyseal, supracondylar, femoral fractures, outcomes, malrotation, malreduction, nonunion, malalignment

\section{Introduction}

After surgical treatment of femoral diaphyseal and supracondylar fractures early and late complications can occur ${ }^{1,2}$.

Among early complications the most important are shock, as up to two liters of blood can be lost within the soft tissues even in closed fracture; infection, reported in 3-5\% of cases; deep venous thromboembolism (1-10\%) and ARDS or fat embolism, (up to $11 \%$ in case of poly-injured patients with associated lung trauma) ${ }^{3}$. The incidence of all these complications can be reduced by correct assessment of the injured patient, fluid and blood resuscitation, surgical timing and prompt antibiotic and thromboembolic prophylaxis.

The most important late complications are malreduction (mainly malrotation and varus/valgus deformities), and nounions ${ }^{4-7}$.

The authors believe that in most of cases complications are determined by mistakes made during the planning process or during execution of the surgical procedure. A retrospective analysis of complications can help understand the original mistake, making the whole process predictable and the error less likely to occur again.

This article will focus on the most frequent complications determined by improper planning or during the surgical procedure for treatment of femoral shaft/distal epiphysis fractures, providing an analysis of what has gone wrong and why.

\section{Planning errors}

Planning of the surgical procedure is a complex and meticulous process that must take into account the patient's most important features (weight, age, functional demand), attitudes (smoke, alcohol, drug use/abuse), morbidities and medications, and fracture characteristics, like classification, soft tissue involvement and stability. After complete assessment of the fracture, including a thorough imaging study, is completed, a treatment plan can be made. The plan should include how to reduce 


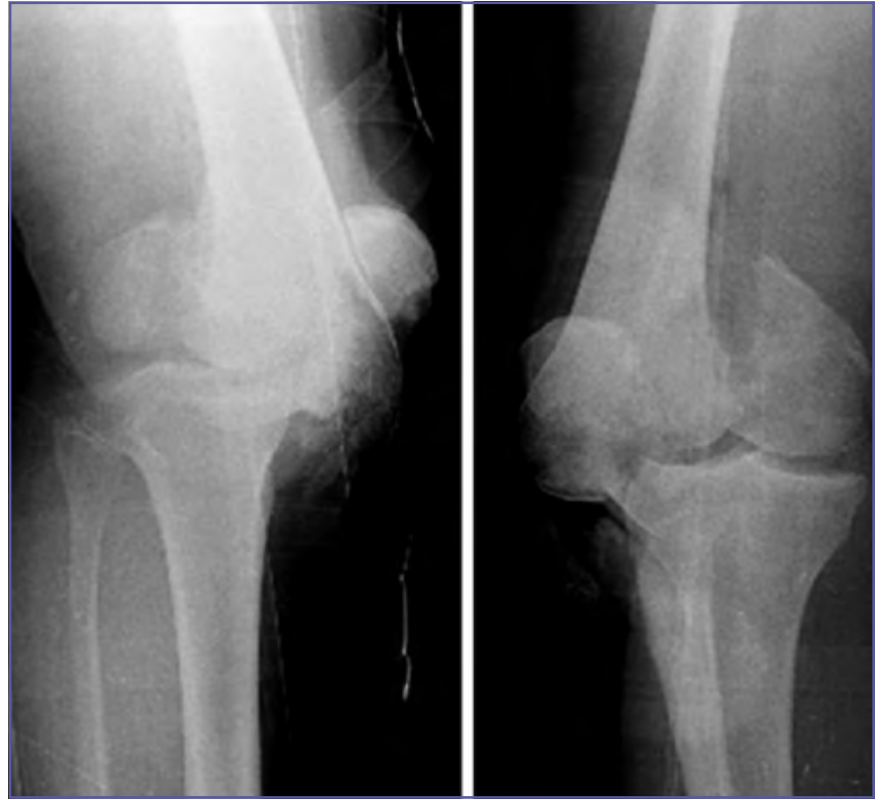

Figure 1. Case 1, ER X-rays: open 3B fracture of distal femur in 69-year-old woman.
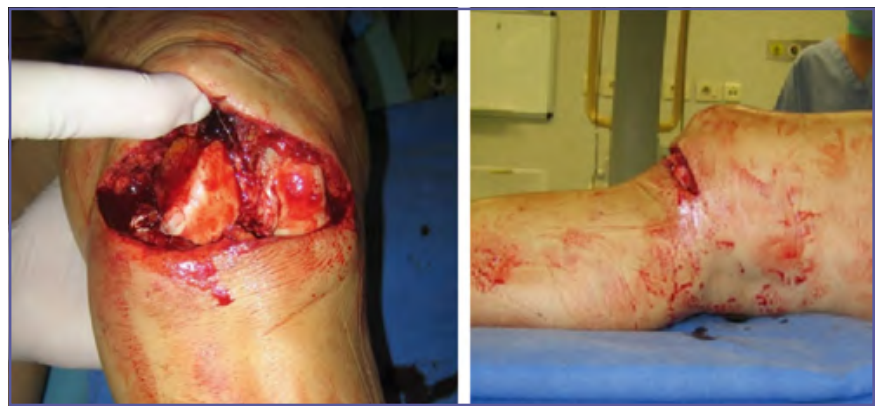

Figure 2. Case 1, clinical photograph: open 3B fracture of distal femur in 69-year-old woman.

the fracture, through which approach and the best fixation device to use. A good pre-operative planning should also consider the possibility of a "B" option.

Failure to do so can lead to intraoperative difficulties. An example of how surgery without planning can go wrong is shown in Case 1. It is the case of a 69-year-old woman who sustained an open 3B fracture of the distal femur (Figs. 1, 2). In emergency she was treated by washing, debridement and fracture stabilization with a spanning external fixator. At day 4 after the trauma without having done any more diagnostic exams the patient underwent definitive surgery, consisting in external fixator removal and fracture reduction and fixation with screws and a LCP-df plate on the medial side. The post-operative X-rays are shown in Figure 3. She was reviewed clinically and radiographically at one, three and six months: she always complained of a valgus knee and mild pain during walking. At 14 months she experienced a lateral patellar dislocation. On that occasion a CT scan was taken that revealed an unhealed fracture of an unidentified Hoffa fracture of the lateral femoral condyle (Fig. 4). The fracture has never been fixed because its pattern was not clearly shown on the almost inexistent pre-operative diagnostics. In the same way, progressive dislocation of the postero-lateral fragment was never detected (even if evident) on the follow-up $\mathrm{X}$-rays. A conservative treatment was attempted, but progressive valgus, recurrent lateral patellar dislocation and severe knee pain made total knee replacement necessary (Fig. 5).

Sometimes an incorrect plan arises from insufficient patient examination or from a superficial knowledge of the treatment device used. This is the case of a 78-year-old woman (case 2) with a fracture of femoral diaphysis (Fig. 6). She underwent surgery within few hours from admission to the ED. The operating surgeon without any doubt believed in the algorithm that all femoral fractures are best treated with a nail and operated on her accordingly (Fig. 7). Two weeks after the operation the patient was still unable to bear weight on the affected limb and had to sit in a chair because of pain. New radiographs were taken (Fig. 8) revealing implant mobilization. The patient underwent a second procedure to remove the nail and to stabilize the fracture with a LCP plate and angular stability locking screws, which has been known to be an implant designed especially for osteoporotic bone. The patient eventually healed after 6 months without any additional problems (Fig. 9). Good pre-operative planning should take into account the patient's characteristics and the properties of the instruments used. In

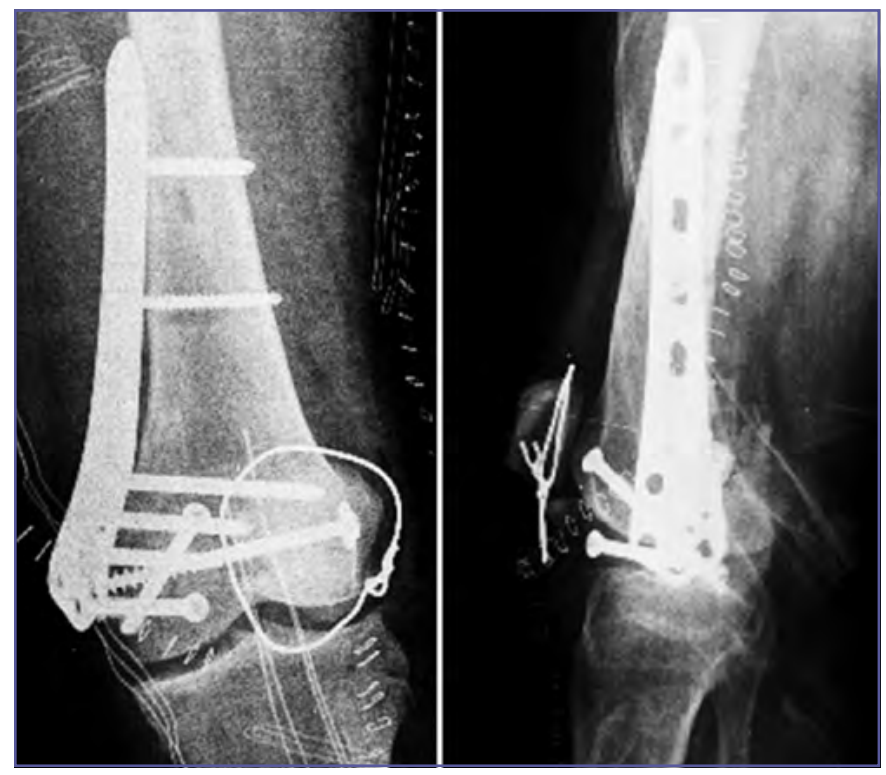

Figure 3. Case 1, post-operative X-rays: definitive surgery with screws and medial LCP-df plate without previous $\mathrm{CT}$ scan planning. 


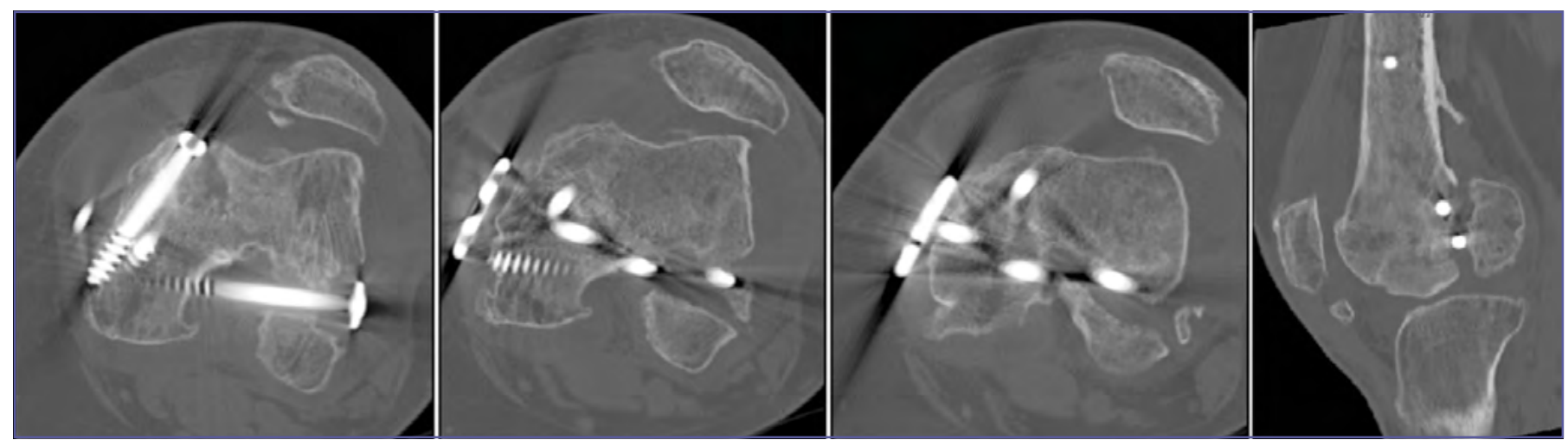

Figure 4. Case 1, CT scan after lateral patellar dislocation: nonunion of a unidentified Hoffa fracture of the lateral femoral condyle.

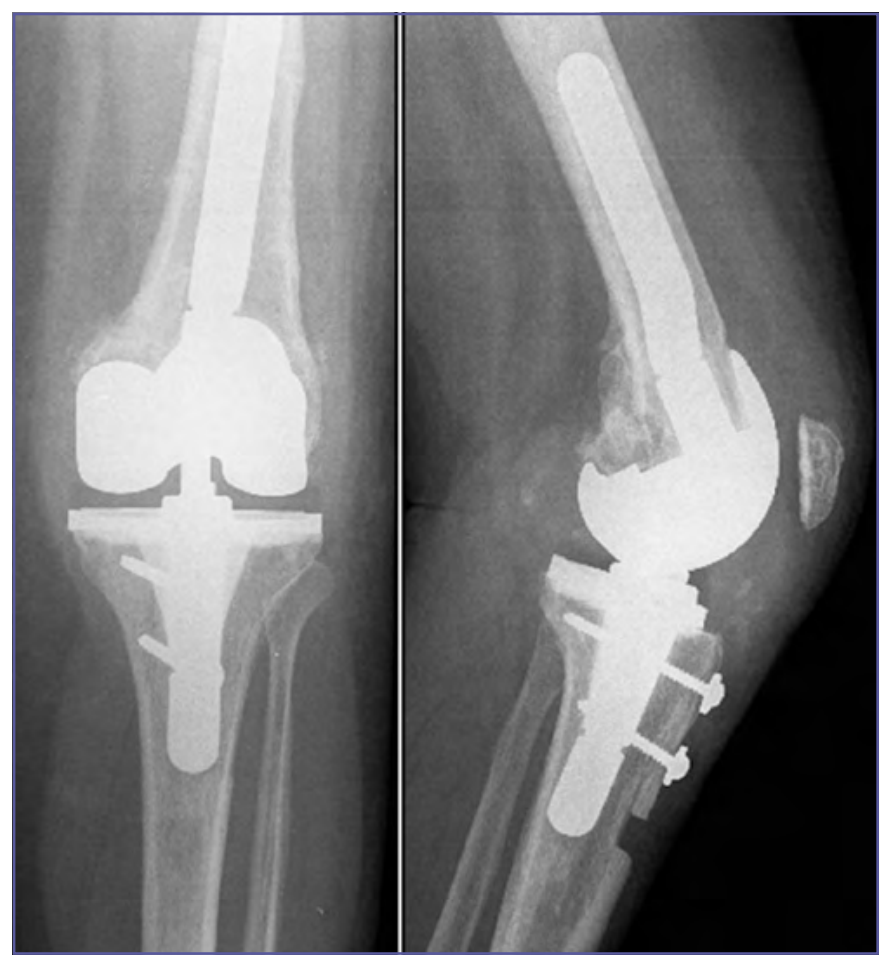

Figure 5. Case 1, total knee replacement as final solution.

this specific case, the wrong belief that a nail is always the best solution to fix a femoral diaphyseal fracture was the basis for failure. In an osteoporotic patient, the femur has a wide canal and a weak bone. This means that an intramedullary nail (even the biggest one provided) will not be able to fill in it completely and the locking screws will not have enough purchase in the thin metaphyseal cortices. This kind of osteosynthesis will not be stable enough to let the fracture heal. Conversely, a locking plate anatomically shaped for distal femoral fractures is, especially in this pattern of fracture with a short distal fragment, the best solution, allowing to place a greater number of more distal screws in the short fragment and giving the right stiffness that the fracture needs to heal. This is to say that not all the femoral fractures are best treated by nails, and the best treatment should be decided at the end of an accurate pre-operative plan.

\section{Technical errors}

Technical errors occur when the surgical plan has been made, but there are flaws in it (concept error) or when the surgical procedure is not performed in accordingly to the plan made (manual error) ${ }^{8}$. Typically concept errors are the cause of inadequate fixations which lead to healing difficulties (delayed unions) or inability

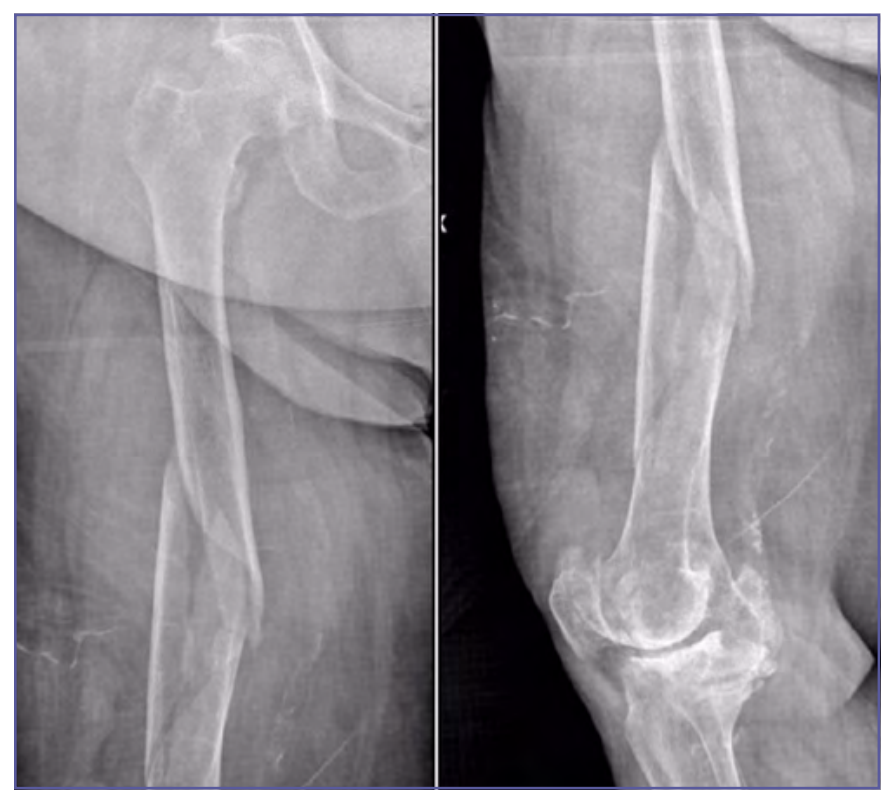

Figure 6. Case 2, ER X-rays: femoral diaphyseal fracture in a 78-year-old woman. 

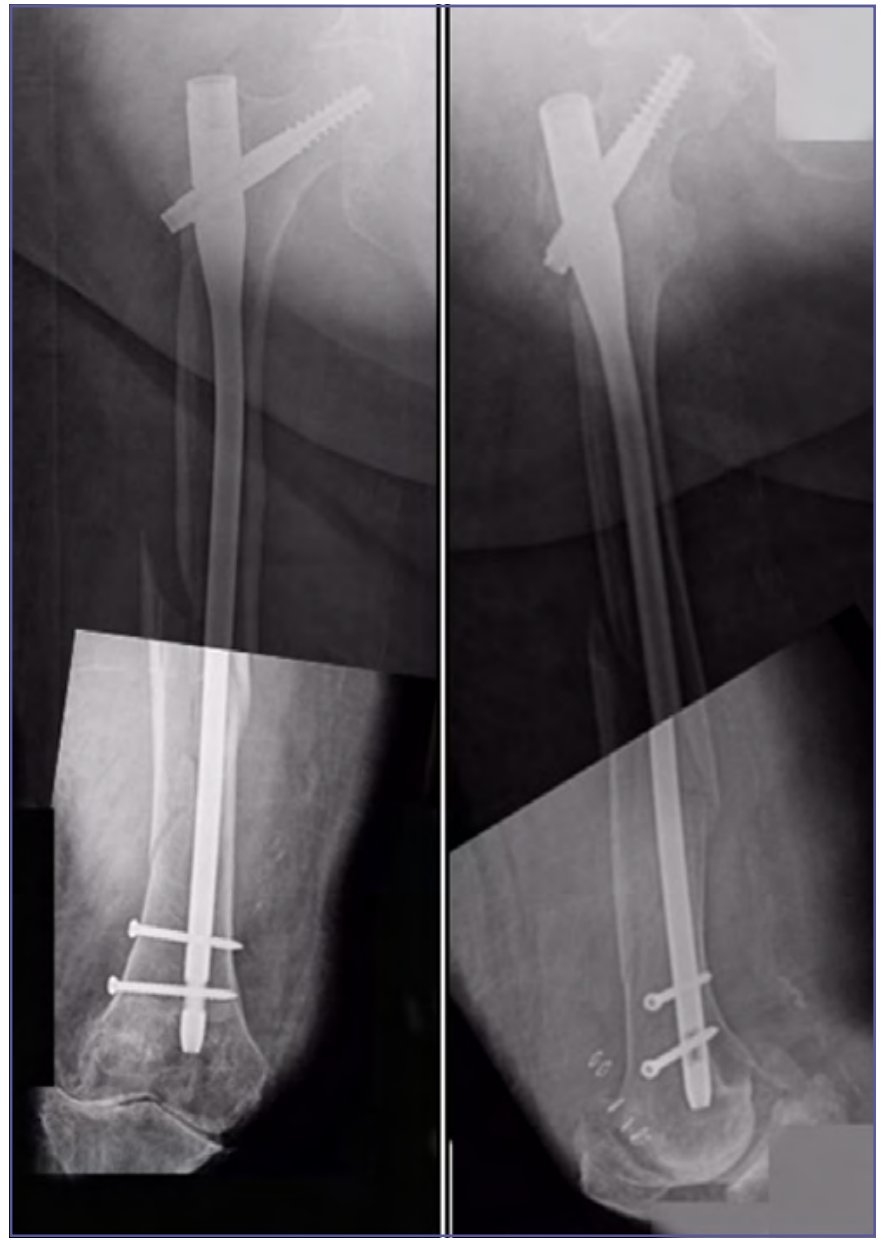

Figure 7. Case 2, post-operative X-rays: surgery within few hours, nailing.

(nonunions) ${ }^{9}$. It is well known that bone healing is a dynamic process that needs an adequate biomechanical environment and a vital bone. Failure to give appropriate stability to the fracture or excessively damaging soft tissues and periosteoum can cause problems in healing.

Nonunion is reported in up to $5 \%$ of cases after nailing and in up to $15 \%$ after plating fixation of diaphyseal and supracondylar fracture ${ }^{10,11}$. There are patient-related factors that affect negatively the development of nonunion, such as cigarette smoking and use of NSAIDs. Good practice should be to put efforts in limiting such factors, making patients aware. Apart from this, nonunion can be caused by one or more mistakes made during surgery such as too much or too little implant stiffness, unbalanced osteosynthesis or, worse, an open or too aggressive surgery in cases where blood supply must be preserved.

An example of a concept error is well represented by the case 3. A 53-year-old male sustained an AO-OTI $33 \mathrm{C} 2$ fracture of

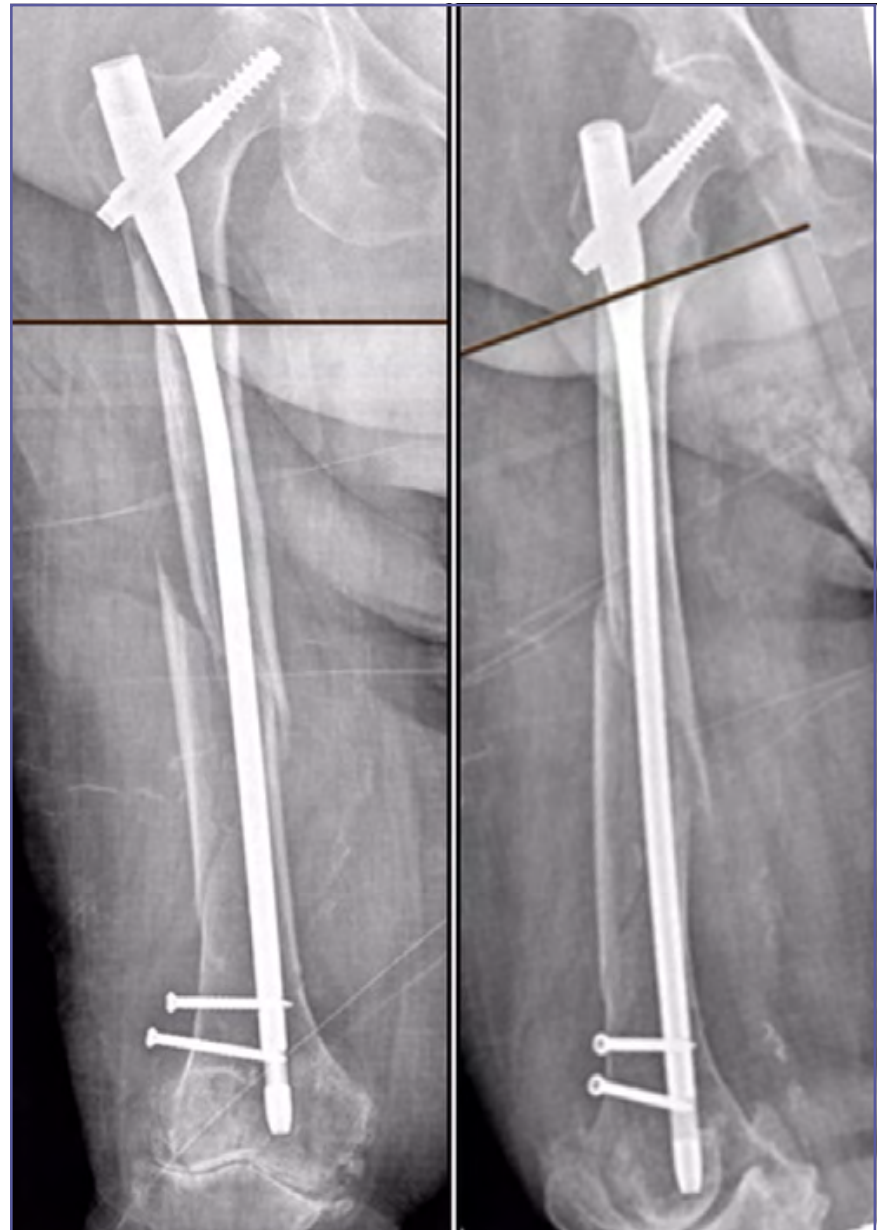

Figure 8. Case 2, X-ray at 15 days: patient unable to bear weight, pain. Radiographs show mobilization of the implant.

the femur. He had been treated by open reduction and fixation with DCS plate and screws (Fig. 10). He referred to our hospital after 18 months from surgery with increasing pain and severe gait limitation. $\mathrm{X}$ rays showed fixation failure and varus collapse of the fracture (Fig. 11).

Analysis of the fixation showed that the surgeon made an open reduction of a multifragmentary fracture in order to obtain alignment of the fragments and searched for a rigid fixation with a short and strong plate filled with screws. This plan has two main flaws. From a biological point of view, a multifragmentary fracture needs blood supply preservation to heal with callus formation, while biomechanically a less rigid fixation is required to give micro-motion which is needed to heal. Open surgery means soft tissue damage and impaired healing potential. On the other hand, if a long healing time is expected a short and rigid plate is less likely to endure until consolidation. Moreover, in this particular type of fracture a medial support is needed to counteract varus deforming forces. 
In this case, a correct plan should have been a closed reduction and fixation with a long lateral fixed angle anatomical distal femoral plate, preserving the most the fragmented area, and a medial less invasive plate. Sometimes even in the presence of a good plan we can fail to put it in practice. In doing so we are making a manual error. Manual errors usually consist in the inability of obtaining a good reduction, determining malalignment, or in bad soft tissues and bone management, leading to healing difficulties, such as surgical site infections or atrophic nonunion. Malreduction is the most frequent manual error. Malrotation is reported in up to $15 \%$ of the cases of comminuted fractures

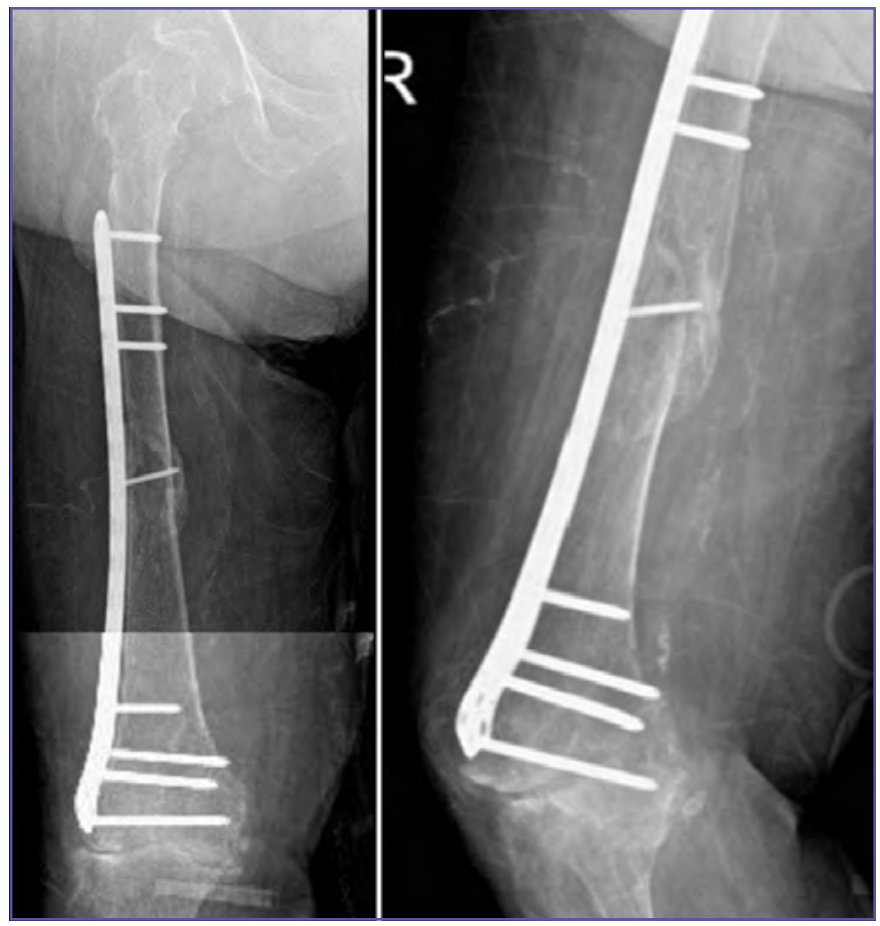

Figure 9. Case 2, X-rays at 6 months after revision surgery.

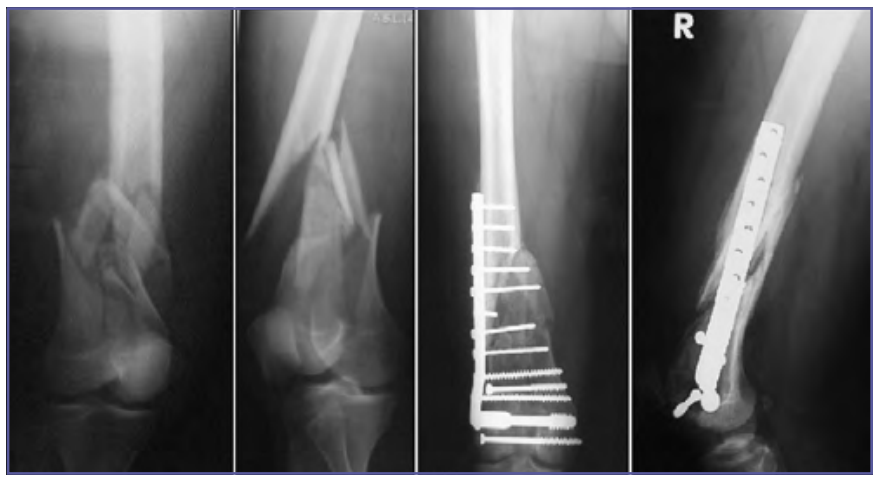

Figure 10. Case 3, ER and post-operative X-rays: 53-year-old male with AO OTI $33 \mathrm{C} 2$ femoral fracture. Definitive surgery with DCS plate.

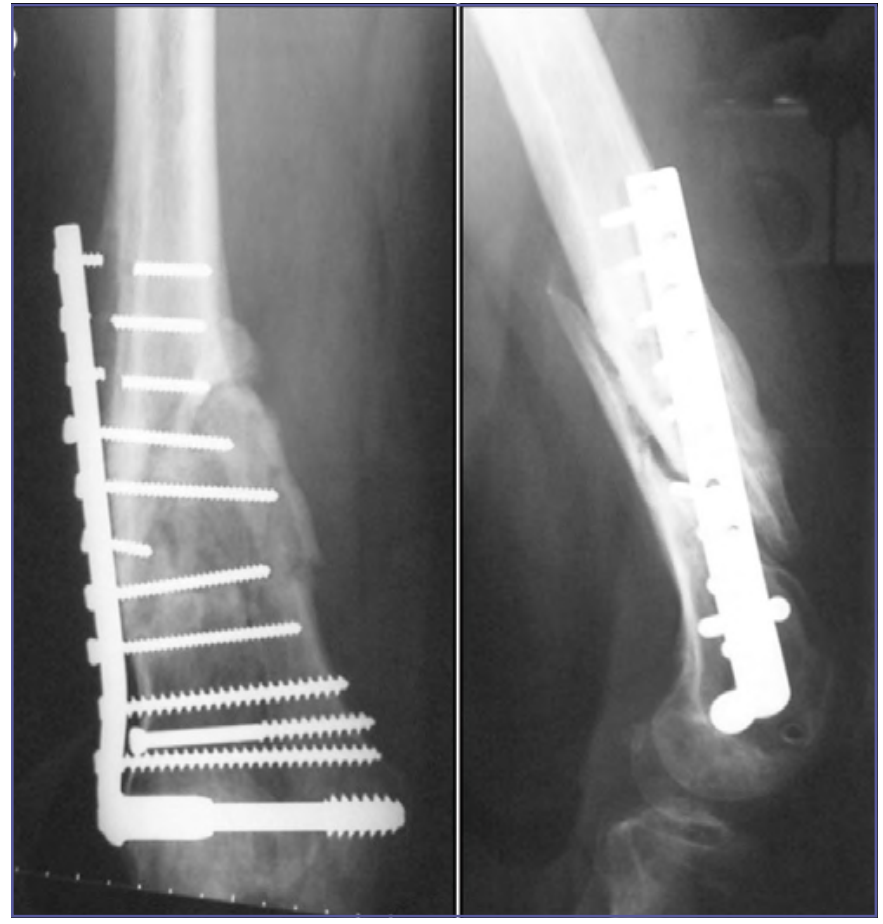

Figure 11. Case 3, X-rays at 18 months: worsening pain and severe gait limitation. Radiographs show fixation failure and varus collapse of the fracture.

of the femoral diaphysis, while varus and valgus deformity are more frequent in distal diaphyseal and supracondylar fractures and are equally reported when using a nail or a plate.

When a nail is used, the factor influencing most the inability to obtain a good reduction is the discrepancy between the diameter of the nail and the intramedullary canal. Many solutions have been proposed to solve this problem. One of the most effective is the use of so-called "poller" screws: free screws to put where the metaphysis is larger in order to create a narrow canal where the nail is guided. The use of one or more "poller" screws in different planes has been reported to be effective in reducing the incidence of axial malalignment in nailing distal femoral fractures.

On the other hand, when plates are used to treat supracondylar fractures axial deformities can be observed due to the difficulty of visualize and control the distal fragment, mainly when a MIPO technique is used. Careful use of the c-arm, meticulous technique and knowledge of the many different direct and indirect reduction tools can aid in reducing the rate of this complication.

An example: a 45-year-old female, victim of a car accident, referred to our ER for a closed diaphyseal femoral fracture with a wedge fragment (AO-OTA 32 B, Fig. 12). She had no major comorbidities and she was operated on the same day with a long locked femoral nail (Fig. 13). At 7 months the fracture had 


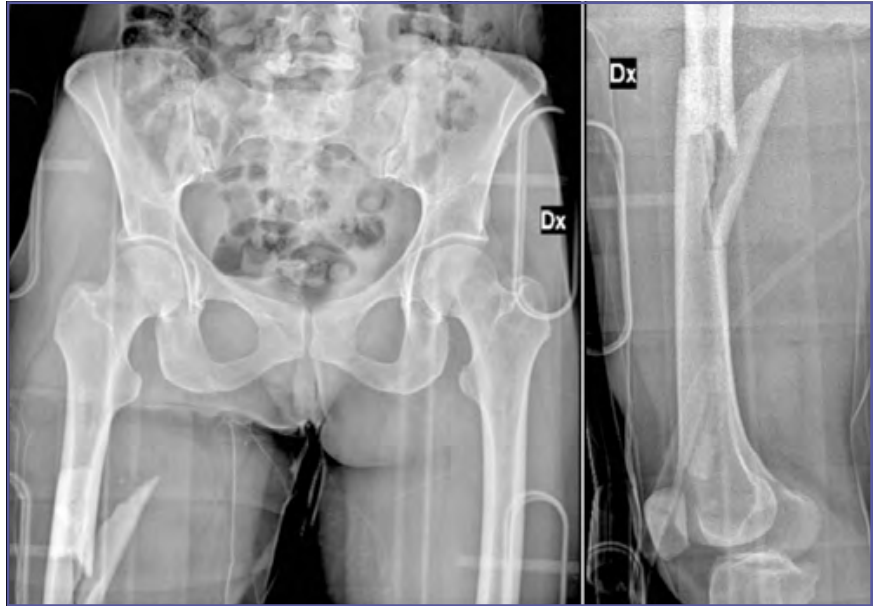

Figure 12. Case 4, AO-OTA 32 B. Diaphyseal femoral fracture with wedge fragment in 45-year-old female.
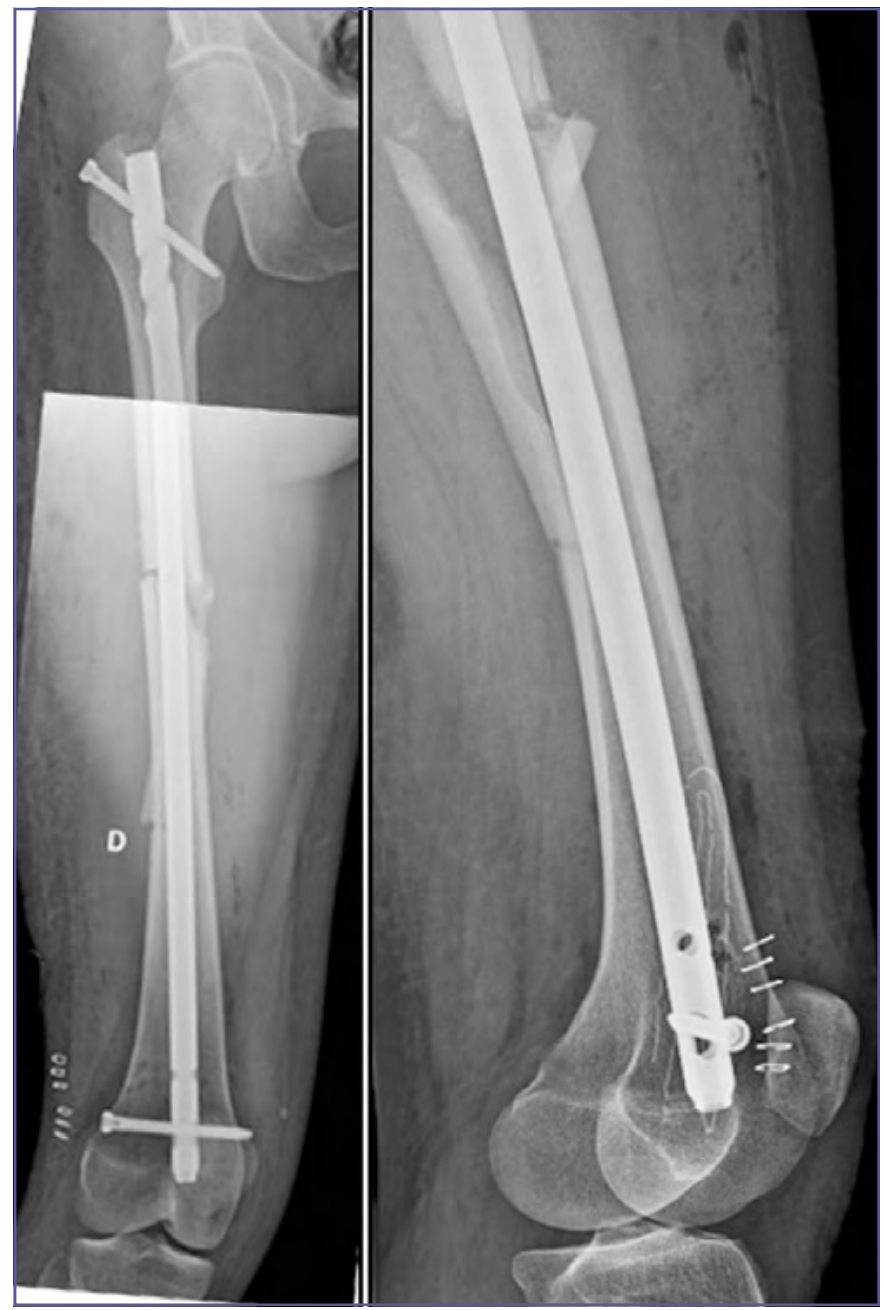

Figure 13. Case 4, post-operative X-rays.not completely

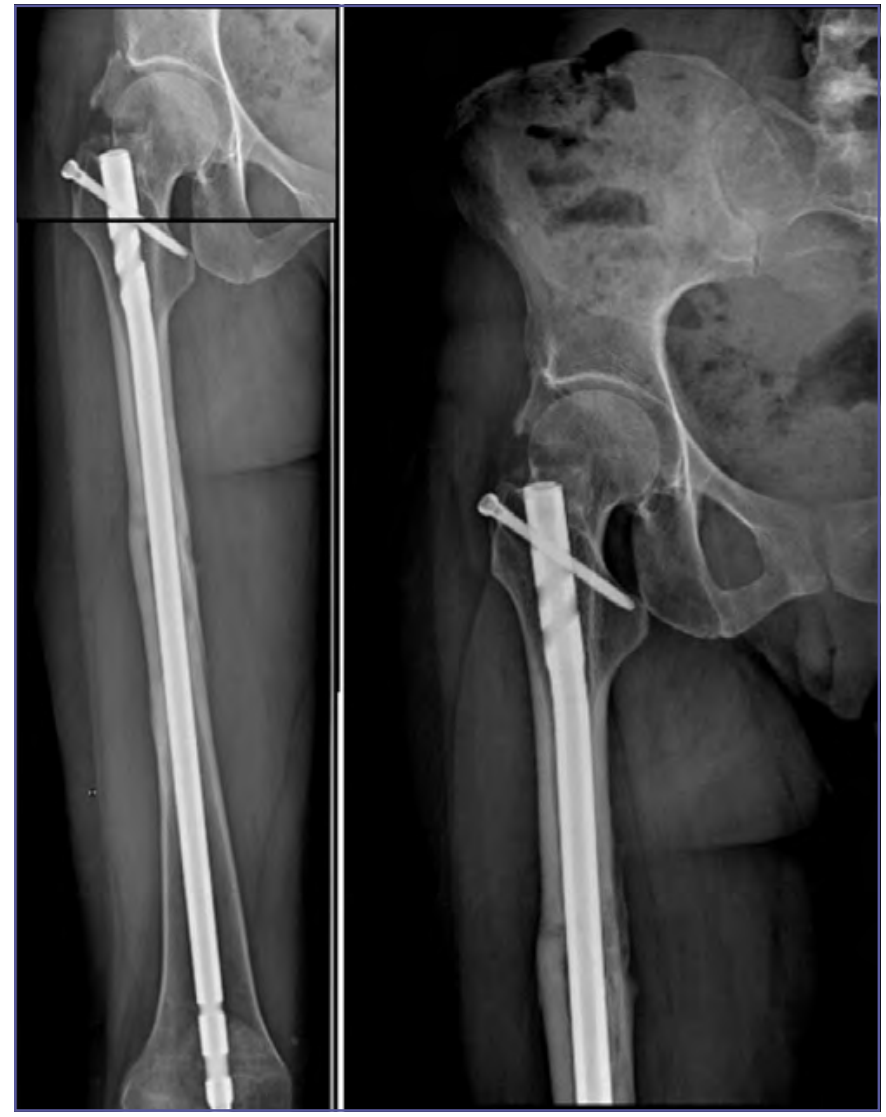

Figure 14. Case 4, 10-month follow-up shows a healed fracture.

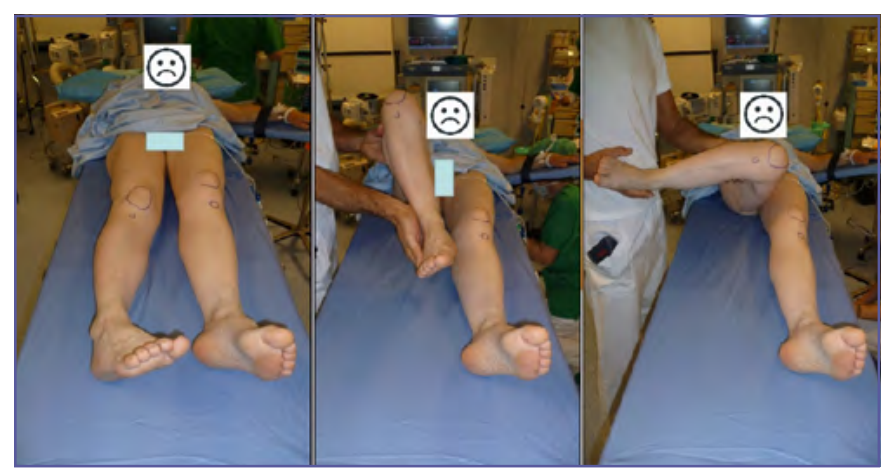

Figure 15. Case 4, clinical examination shows $50^{\circ}$ hip internal rotation.

healed, and so nail dynamization was undertaken with complete bone healing within 10 more months (Fig. 14).

At two years follow-up, the patient had no pain but complained about gait difficulties during walking. Clinical examination and CT showed a intrarotation of $50^{\circ}$ of the affected limb (Fig. 15). Surgical correction of the deformity was required: it consisted 


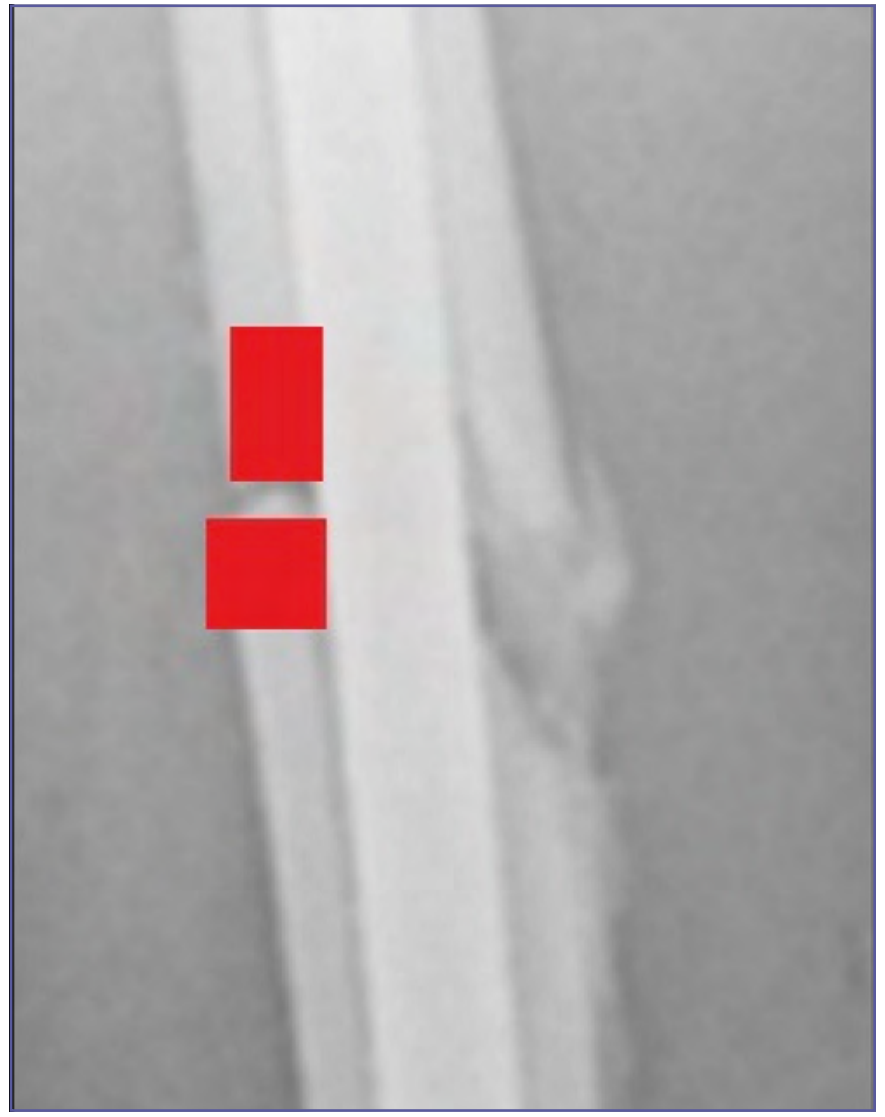

Figure 16. Case 4, importance of checking cortical diameters during reduction phase.

in nail removal, femoral osteotomy, correction of the deformity and a new fixation with a LCP 4,5 broad plate.

Although rotational assessment during femoral nailing can be challenging, accurate patient positioning and careful visualization of the entire femur during surgery, showing the hip the knee and the fracture site could had avoided the problem (Fig. 16).

Moreover, it is always a good habit to compare clinically the rotation of the lower limbs after surgery and to visualize the post-operative X-rays for early detection of such malalignment. In this particular case, none of the above were done, leaving the technical error unseen and leading to a late correction of the deformity.

\section{Conclusions}

The treatment of femoral diaphysis and distal epiphysis fractures is challenging. Many complications have been reported such as malunion, nonunion or soft tissue problems. In most cases an accurate analysis of the entire history of the fracture can put in evidence one or more mistakes made during the planning phase or during the practical phase of the surgical act. Understanding the reasons and modalities that lead to the onset of the problem can help the surgeon in preventing to repeat the same mistake in the future.

\section{References}

1 Koso RE, Terhoeve C, Steen RG, et al. Healing, nonunion, and re-operation after internal fixation of diaphyseal and distal femoral fractures: a systematic review and meta-analysis. Int Orthop 2018;42:2675-83. https://doi.org/10.1007/s00264-018-3864-4

2 Rupp M, Biehl C, Budak M, et al. Diaphyseal long bone nonunions - types, aetiology, economics, and treatment recommendations. Int Orthop 2017;42:247-58. https://doi.org/10.1007/s00264-017-3734-5

3 Canadian Orthopaedic Trauma Society. Reamed versus unreamed intramedullary nailing of the femur: comparison of the rate of ards in multiple injured patients. J Orthop Trauma 2006;20:384-7. https://doi.org/10.1097/00005131-200607000-00003

4 Guerado E, Bertrand ML. Malalignment in intramedullary nailing. How to achieve and to maintain correct reduction? Injury 2017;48:S30-4. https://doi.org/10.1016/j.injury.2017.04.027

5 Taitsman LA, Lynch JR, Agel J, et al. Risk factors for femoral nonunion after femoral shaft fracture. J Trauma 2009;67:1389-92. https://doi.org/10.1097/ta.0b013e318182afd0

6 Giannoudis PV, MacDonald DA, Matthews SJ, et al. Nonunion of the femoral diaphysis. J Bone Joint Surg Br 2000;82-B:655-8. https://doi.org/10.1302/0301-620x.82b5.0820655

7 Dagneaux L, Allal R, Pithioux M, et al. Femoral malrotation from diaphyseal fractures results in changes in patellofemoral alignment and higher patellofemoral stress from a finite element model study. Knee 2018;25:807-13; https://doi.org/10.1016/j.knee.2018.06.008

8 Neumann MV, Südkamp NP, Strohm PC. Management of femoral shaft fractures. Acta Chir Orthop Traumatol Cech 2015;82:22-32.

9 Wiss DA, Fleming CH, Matta JM, et al. Comminuted and rotationally unstable fractures of the femur treated with an interlocking nail. Clin Orthop Relat Res 1986;212:35-47.

10 Ricci WM, Bellabarba C, Lewis R, et al. Angular malalignment after intramedullary nailing of femoral shaft fractures. J Orthop Trauma 2001;15:90-5.

11 Buckley R, Mohanty K, Malish D. Lower limb malrotation following MIPO technique of distal femoral and proximal tibial fractures. Injury 2011;42:194-9. https://doi.org/10.1016/j.injury.2010.08.024 